\title{
Estimation of the parameters of barbell' lifting law of motion
}

\author{
Mečislovas Mariūnas ${ }^{1}$, Julius Griškevičius ${ }^{2}$, Gintaras Jonaitis ${ }^{3}$ \\ 1,2,3 Vilnius Gediminas Technical University, Lithuania \\ E-mails: ${ }^{1}$ mecislovas.mariunas@vgtu.lt (corresponding author), ${ }^{2}$ julius.griskevicius@vgtu.lt, \\ 3 gintaras.jonaitis@vgtu.lt \\ (Received 1 July 2016; accepted 16 August 2016)
}

\begin{abstract}
Characteristic points of the law of motion were determined based on the results of experimental study on the velocities and accelerations of the lifted barbell. Relationship between the height of the athlete, lifted weight, maximal velocity and acceleration was estimated analytically. It was shown, that at the initial time moment the athlete must apply approximately 1,5 times larger force than lifted weight. The values of the parameters of the barbell' law of motion and lifting force approximate law of change were estimated.
\end{abstract}

Keywords: dynamics, maximal velocities, accelerations, parameters of motion, maximal force, law of motion, height, weight, mass.

\section{Introduction}

The biomechanical analysis of the motion of bar in weightlifting was analysed in various scientific research works $[1,2,3,4,5,6,7,8,9,10,11]$. The trajectory of barbell motion and accelerations and velocities were measured. Experimentally estimated maximal velocities of the barbell for the experienced athletes and it is equal to $\sim 1.0 \mathrm{~m} / \mathrm{s}$. In the study [11] it was explained, that maximal acceleration for different level athletes can vary from 2.8 to $5.13 \mathrm{~m} / \mathrm{s}^{2}$. When analysing time dependency of the motion velocity during the snatch of highly skilled athletes, it was estimated, that it varies continuously, almost symmetrically and without sudden oscillations [4]. The magnitude of the lifted weight depends on the height of the athlete [5]. The taller the athlete, the lower weight he is able to lift. Some research works present studies on the kinetostatics of the barbell using Newton law of motion. However, there is lack of works devoted to development of analytical estimation of the dependency of the maximal velocity on the lifted height and needed acceleration to guarantee maximal velocity.

Therefore, the goal of present research is, based on the experimental results, estimate relationship between the height of the athlete, lifted weight, maximal velocity and acceleration. Also, determine characteristic points of law of motion of the barbell and values of parameters in those points.

\section{Methods}

Research was carried out in the weightlifting gym of the Vilnius University "Health and sport centre" under the guidance of the weightlifting coach. Total six athletes were recruited for the study: three beginners and three advanced weightlifters. The barbell's weight was varying from 60 to $80 \mathrm{~kg}$. The height of the athletes was $1.62-1.86 \mathrm{~m}$. The barbell's lifts were the snatch and the clean and jerk. Inertial sensors with $\pm 1.5 \mathrm{~g}-6 \mathrm{~g}$ Three Axis low-g micromachined accelerometer were fixed on the hand and forearms of the weightlifters and one on the barbell. Weightlifters were instructed to perform snatch and clean and jerk lifting techniques. All data was registered and stored on the PC. 


\section{Results}

Some results of the experimental measurements are shown in Table 1 and Fig. 1 and Fig. 2. Table 1 presents athlete-characteristic data of the barbells' velocity and acceleration. Analysis of the influence of the height to maximal velocity $v_{\max }$ showed that the taller athlete must generate larger maximal velocity in order to lift the same weight and they can lift lower maximal weight than smaller athlete can. This was estimated also by Jaya et al in [5].

Table 1. Details about the weightlifter

\begin{tabular}{|c|c|c|c|c|c|c|c|c|c|}
\hline $\begin{array}{c}\text { Subject } \\
\mathrm{N}\end{array}$ & $\begin{array}{c}\text { Height } \\
h, \mathrm{~cm}\end{array}$ & Age & $\begin{array}{c}\text { Human } \\
\text { weight, } \\
\mathrm{kg}\end{array}$ & $\begin{array}{c}\text { Lifted } \\
\text { weight } \\
Q, \mathrm{~kg}\end{array}$ & $\begin{array}{c}a_{m,}, \\
\mathrm{~m} / \mathrm{s}^{2}\end{array}$ & $\begin{array}{c}t, \mathrm{~s} \\
\text { when } \\
a_{m} \approx 0\end{array}$ & $\begin{array}{c}v_{\max }, \\
\mathrm{m} / \mathrm{s}\end{array}$ & $h / v_{\max }$ & $\begin{array}{c}t, \mathrm{~s} \\
\text { when } \\
v=v_{\max }\end{array}$ \\
\hline $1 \mathrm{n}$ & 186.0 & 23 & 74 & 70 & 3.85 & 0.6 & 1.50 & 124.00 & 0.6 \\
\hline $\mathrm{n} \mathrm{n}$ & 178.5 & 22 & 76 & 70 & 4.05 & 0.55 & 1.46 & 122.26 & 0.55 \\
\hline $3 \mathrm{n}$ & 169.5 & 24 & 80 & 70 & 3.68 & 0.6 & 1.42 & 119.37 & 0.6 \\
\hline $4 \mathrm{p}$ & 162.0 & 22 & 73 & 70 & 3.33 & 0.55 & 1.05 & 154.28 & 0.55 \\
\hline $5 \mathrm{p}$ & 175.0 & 21 & 75 & 70 & 3.58 & 0.5 & 1.16 & 150.87 & 0.5 \\
\hline $6 \mathrm{p}$ & 172.5 & 23 & 77 & 70 & 3.16 & 0.55 & 1.10 & 156.82 & 0.55 \\
\hline
\end{tabular}

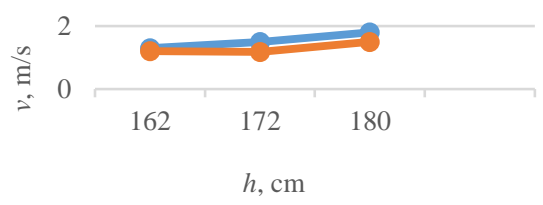

Fig. 1. Influence of the height to barbell $(70 \mathrm{~kg})$ velocity: blue - one subject, orange - averaged data of 3 subjects

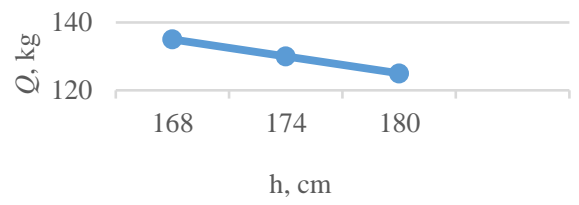

Fig. 2. The influence of height to the lifted weight, according to [5]

From the Fig. 1 it can be noted that there is non-linear relationship between maximal velocity and athlete height, because $v_{1} / h_{1}<v_{2} / h_{2}<v_{3} / h_{3}$ and it can be expressed as second order polynomial.

Analysing changes tendencies of barbell's velocities and accelerations during snatch and clean and jerk it was noted that maximal velocity $1.1-1.75 \mathrm{~m} / \mathrm{s}$ is reached after $0.9-1.2 \mathrm{~s}$, while maximal acceleration $3.16-3.85 \mathrm{~m} / \mathrm{s}^{2}$ is reached after $0.21-0.3 \mathrm{~s}$ (Table 1). Their velocity is increasing continuously and after it reaches maximal value starts to decrease (Fig. 3, 4). Fig. 3 shows graph of the $70 \mathrm{~kg}$ weight barbell clean and jerk, the height of the athlete $172 \mathrm{~cm}$, maximal velocity $v_{\max } \approx 1.75 \mathrm{~m} / \mathrm{s}$, and Fig. 4 - during snatch of the same weight, $h=162 \mathrm{~cm}$, and velocity is only $v_{\max } \approx 1.1 \mathrm{~m} / \mathrm{s}$. While analysing velocities and accelerations of barbell's motion weaknesses of the athlete can be noted and one can suggest how to improve.

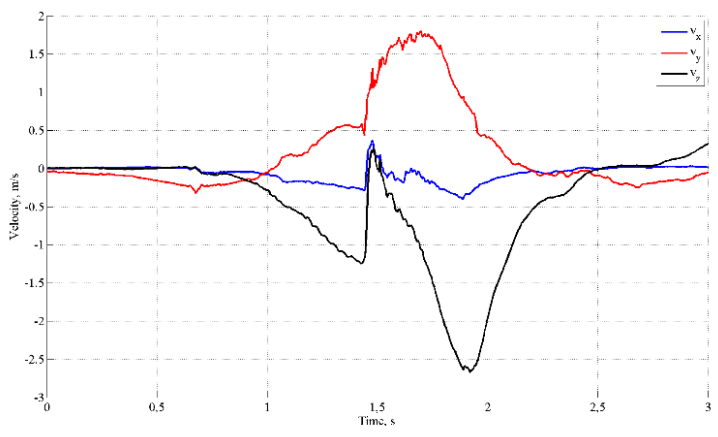

Fig. 3. Barbell' lifting velocity of beginner athlete 


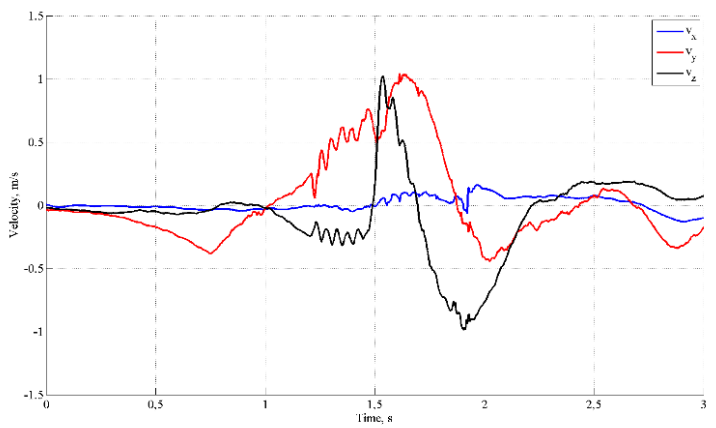

Fig. 4. Barbell' lifting velocity of advanced athlete

The characteristic points of the barbell law of motion and their values were estimated: first when $t=0$, the velocity $v(t)=0$, acceleration $a(t)=0$ and the lifting height of the barbell $h(t)=0$; second - when $a(t)=a(t)_{\max }, 0<t<T$ and $v(t) \neq 0$; third - when $a(t) \approx 0, F(t) \approx 0$ and $0 \leq t \leq T / 4$, the value of the velocity is maximal $v(t) \approx v_{m}$ and $0<h(t)<h_{m}$ and, fourth - when $t=T, a(t)=0$, $v(t)=0$ and $h(t)=h_{m}$, (where $T$ - barbell lifting time; $h_{m}$ - barbell lifting height).

The dynamics of the barbell motion will be investigated further after the evaluation of values of characteristic points of barbell's law of motion. Barbell's motion in the direction of $0 x$ axis under action of external force can be described as:

$m \ddot{x}(t)+k \dot{x}(t)^{2}+Q=F(t)$, when $a(t) \neq 0$ and $\mathrm{t}>0, F(t)=F(t)_{d}+Q$,

where $\ddot{x}(t)$ is second time derivative, $\dot{x}(t)$ - first time derivative; $F(t)$ - force applied to the barbell; $k$-coefficient of the environmental drag; $m$ - barbell's mass, $F(t)_{d}$ - variable part of the force, $Q$ - barbell's weight.

When $a(t) \approx 0$ and $0<t<T$, then $F(t) \approx 0$ and equation (1) becomes:

$m \ddot{x}(t)+k \dot{x}(t)^{2}+Q=0$.

Equation (2) shows that when the barbell is under action of $F(t) \approx 0$, it will move due to inertia. Based on the barbell velocity (Fig. 3, 4) and acceleration graphs, time varying functions of the kinematic barbell's parameters can be expressed using functions of Fourier series and taking only first elements the solution of equation (1) is:

$$
\begin{aligned}
& F(t)_{d}=\frac{\pi m}{T} v_{\max } \cos (\omega t-\varphi)+k\left[v_{\max } \sin (\omega t-\varphi)\right]^{2}, \text { when } 0 \leq t \leq \frac{T}{4}, F(t)=F(t)_{d}+Q ; \\
& F(t)_{d \max } \approx \frac{\pi m v_{\max }}{T} \text { and } a_{\max } \approx \frac{\pi v_{\max }}{T},
\end{aligned}
$$

where $\omega$ - angular velocity; $\varphi$ - initial phase.

When $\omega t-\varphi=0$, then $\sin (\omega t-\varphi)=0$. In such way, the magnitude of the force when lifting the weight at initial time moment would be described according to the first member of (3) expression. When $t \approx T / 4$, then $\cos (\omega t-\varphi)=0$ and only second member remains in (3) expression. Therefore, the force applied by the skilled athlete at initial time moment and during the lifting should vary approximately according to cosine law. 
When the value of the acceleration $a(t) \approx 0,0 \leq t \leq T$ and $v(t) \neq 0$, the lifted barbell moves due to inertia (2). It is important to provide required velocity to the barbell in order to reach desired lifting height. Evaluating estimated characteristic points of the law of motion, dependencies of the lifted height, barbell's weight and maximal velocity were obtained analytically:

$$
h_{0} \leq \frac{k}{2 g m} \ln \left[\frac{\left(1+\frac{k}{g m} v_{m}^{2}\right)}{\left(1+\frac{k}{g m} \dot{h}(t)^{2}\right)}\right] \text {, }
$$

where $g$ - gravitational acceleration; $v_{m}$ - maximal velocity; $h_{0}$ - height of the barbell's due to inertia, i.e. when $a(t) \approx 0$, and $v_{m} \neq 0 ; \dot{h}(t)^{2}$ - derivative squared.

Assuming, that the environmental drag during the lifting of the barbell is low, the approximate solution of (2) equation can be written as follows:

$$
h_{0} \leq \frac{v_{m}^{2}}{2 g} \text { or } v_{m}>\sqrt{2 h_{0} g}
$$

The last expression (5) shows the approximate dependency of barbell's maximal velocity on the lifting height when $a(t) \approx 0$ and $v_{m} \neq 0$, i.e. what height it will reach due to inertia. Calculations performed according to (3) and using data from Table 1 has showed that the barbell must have reached approximately $114.7 \mathrm{~mm}$ due to inertia for the taller athlete $(h=186.0 \mathrm{~cm})$, while in case of the smaller athlete $(h=162.0 \mathrm{~cm})-56.2 \mathrm{~mm}$. Therefore, for taller athlete barbell should have lift 2 times more. The larger the athlete, the larger must be the height $h_{0}$ and therefore the higher velocity will be required for covering such a distance due to inertia. Which in turn means, that taller athlete must apply higher force to the barbell than the smaller athlete must or he will lift the barbell lower. The difference between calculated maximal velocities according to (3) and experimentally estimated (Table 1) is approximately $8-10 \%$. Experimental data also confirms that taller athlete must apply higher force to the same weight.

\section{Conclusions}

After analysis of barbell' motion velocities, acceleration results weightlifters, the relationship between main parameters was determined experimentally and analytically and we can summarize:

- It was shown, that taller athlete must generate larger maximal velocity for lifting of the same weight than the smaller athlete.

- Estimated that the barbell moves not the whole path under action of the external force. When the barbell' acceleration approximately is equating to zero, then action of external force approximately equals to zero, and the velocity is maximum and after this moment barbell is moving further due to the inertia.

- Presented analytical dependencies describe relationship between maximal barbell velocity and covered path length due to inertia, maximum acceleration and applied force at initial time moment.

- At the initial time, the force applied to the barbell must be approximately 1,5 times larger than the barbell's weight and it was estimated that the force magnitude is approximately varying according to a cosine law for advanced athlete. 


\section{References}

[1] Frounfelter, G. 2009. Triple extension: the key to athletic power. NSCA's Performance Training Journal 8(1): 14-15.

[2] Gourgoulis, V., et al. 2009. Unsuccessful vs. successful performance in snatch lifts: a kinematic approach, Journal of strength and conditioning research 23(2): 486-494. http://dx.doi.org/10.1519/JSC.0b013e318196b843

[3] Hedrick, A.; Wada, H. 2008. Weightlifting movements: do the benefits outweigh the risks?, Strength \& Conditioning Journal 30(6): 26-35. http://dx.doi.org/10.1519/SSC.0b013e31818ebc8b

[4] Jones, J. R.; Taylor, J. 2010. The biomechanical analysis of the Olympic snatch lift [online]. Western Oregon University [cited 1 July 2016]. Available from Internet: http://www.wou.edu/ jrjones09/biomechanicsfinalpaper.pdf

[5] Rao, P. J., et al. 2015. Kinematic analysis of snatch technique in weightlifting, International Journal of Law, Education, Social and Sports Studies 2(3): 14-17.

[6] Stone, M. H., et al. 2006. Weightlifting, a brief overview, Strength \& Conditioning Journal 28(1): 5066. http://dx.doi.org/10.1519/1533-4295(2006)28[50:wabo]2.0.co;2

[7] Lloyd, R., et al. 2012. Long-term athletic development and its application to youth weightlifting, Strength and Conditioning Journal 34(4): 1. http://dx.doi.org/10.1519/SSC.0b013e31825ab4bb

[8] Isaka, T.; Okada, J.; Funato, K. 1996. Kinematic analysis of the barbell during the snatch movement of elite Asian weightlifters, Journal of Applied Biomechanics 12(4): 508-518. http://dx.doi.org/10.1123/jab.12.4.508

[9] Kim, Y.-J.; Seo, K.-W. 2002. Ground reaction force of the snatch motion and kinesiological analysis by photography, Journal of Sport biomechanics 12(1): 89-104.

[10] Haug, W. B.; Drinkwater, E. J.; Chapman, D. W. 2015. Learning the hang power clean: kinetic and technical changes in four weightlifting naive athletes, Journal of Strength \& Conditioning Research 29(7): 1766-1779. http://dx.doi.org/10.1519/JSC.0000000000000826

[11] Bauer, T.; Isaac, L. 1996. Biomechanical analysis for the coach of Olympic weight lifting, Journal of Sports Medicine and Physical Fitness 9(4): 263-268. 